\title{
Comparison of effects of direct laryngoscopic and fiberoptic oral endotracheal intubation on intraocular pressure
}

\author{
Yıldırım S. ${ }^{1}$, Ozkan A.S. ${ }^{1}$, Akbas S. ${ }^{1}$, Polat N. ${ }^{2}$, Mutlu K. ${ }^{2}$, Durmus M. ${ }^{1}$ \\ ${ }^{1}$ Inonu University, Dept of Anaesthesiology \& Intensive Care, Malatya, Turkey, \\ ${ }^{2}$ Inonu University, Department of Ophthalmology, Malatya, Turkey
}

Background and Goal of Study: In this study; we aimed to compare the effects of direct laryngoscopic and fiberoptic endotracheal intubation on intraocular pressure in nonophthalmic surgery patients.

Materials and Methods: We obtained confirmation from the Inonu University Medical Faculty Hospital Ethics Committee (2015/134) Total of 54 ASA (American Society of Anesthesiologist) Grade 1-2, Mallampati score 1 or 2 , age between 18 to 65 patient planned to undergo nonophtalmic surgery included to study. Patients with glaucoma, diabetes mellitus, cardiovascular and pulmonary diseases, ASA Grade III and IV, BMI more than 35, difficult intubation, undergo obstetrical surgery and propofol, fentanyl, rocuronium contraindicated were excluded from the study. Patients divided randomly into 2 groups as direct laryngoscopic and fiberoptic intubation group. Patients were preoxigenated with $\% 100 \mathrm{O} 2$ for 3 minutes then anesthesia was induced using propofol $2 \mathrm{mg} / \mathrm{kg}$, fentanyl $1 \mathrm{mcg} / \mathrm{kg}$, and rocuronium $0,5 \mathrm{mg} / \mathrm{kg}$ in both groups. After 3 minutes mask ventilation, patients were intubated (women with No:7-7,5, men with No:8-8,5 intubation tube). SBP, DBP, MAP SpO2, PI recorded and IOP (intraocular pressure) measured by ophthalmologist by tonopen were recorded preinduction (basal), after induction, 1,2,3,5 minutes after intubation, respectively. Period between handling of laryngoscope or fiberoptic device after termination of mask ventilation and obtain end tidal $\mathrm{CO} 2$ was accepted as application time and recorded. Study terminated after 5th minute values taken. conflict of interest is not declared. Results and Discussion: There was no statistically significant difference in distirubiton of sex, weight, age, height, BMI, MPS and ASA between groups. Duration of intubation was statistically significantly longer in FOB group $(\mathrm{p}<0,05)$. Heart rate was statistically significantly higher in DLS group at after induction and 1 minute after intubation compared with FOB group $(p<0,05)$. Statistically significant increase was found in intraocular pressure after 1 minute intubation in DLS group compared with FOB group $(p<0,05)$. Conclusion(s): We concluded that endotracheal intubation by FOB could be more useful with regard to endotracheal intubation by DLS in patients with high IOP patologies due to causing significantly less rise in IOP when performed by experienced anesthesiologist. 